Revista Psicologia: Teoria e Prática, 18(2), I29-I4I. São Paulo, SP, maio-ago. 2016. ISSN I5I6-3687 (impresso), ISSN I980-6906 (on-line). http://dx.doi.org//0.15348//980-6906/psicologia.v18n2p/29-14I. Sistema de avaliação: às cegas por pares (double blind review). Universidade Presbiteriana Mackenzie.

\title{
Fatores de risco e desenvolvimento sociocomunicativo em prematuros ${ }^{1,2}$
}

\author{
Ivete Furtado Ribeiro Caldas ${ }^{3}$ \\ Marilice Fernandes Garotti \\ Aurimery Gomes Chermont \\ Adrine Carvalho dos Santos \\ Universidade Federal do Pará, Belém - PA - Brasil
}

\begin{abstract}
Resumo: Introdução: Neonatos prematuros com baixo peso ao nascer podem apresentar déficits neurológicos e alterações no desenvolvimento sociocomunicativo. Interações iniciais mãe-bebê são importantes para avaliar esse processo de risco, verificado a partir de alterações na comunicação não verbal. Objetivo: verificar a relação entre fatores de risco, sociodemográficos e desenvolvimento sociocomunicativo de prematuros para cada grupo de díades em quatro momentos do desenvolvimento. Método: participaram 18 díades com idade gestacional < 36 6/7 semanas e peso ao nascer $\leq$ 2.500 gramas. As sessões aconteceram aos três, seis, nove e doze meses de vida. Resultados: verificou-se que somente as crianças do grupo MP iniciaram interações triádicas aos nove meses $(M=0,16 ; D P=0,40)$. Habilidade envolvendo pessoa, aos três meses, estava positivamente associada ao peso ao nascer $(0,812 ; p<0,05)$ e ao apgar $5(0,861 ; p<0,05)$. Conclusão: fatores de riscos e sociodemográficos interferem no desenvolvimento sociocomunicativo de prematuros.
\end{abstract}

Palavras-chave: desenvolvimento; interação mãe-bebê; prematuro; fator de risco; comunicativo.

\section{RISK FACTORS AND SOCIAL COMMUNICATIVE DEVELOPMENT OF PRETERMS}

\begin{abstract}
Introduction: Premature infants with low birth weight can have neurologicals deficits and changes in social and communicative development. Early mother-infant interactions are important to evaluate the process of risk, checked from changes in non-verbal communication. Objective: Check the relationship between risk factors, socioeconomic and social communicative development of preterms to each group in four stages of development. Methods: Eigtheen dyads participated with gestational age $<36$ 6/7 weeks and birth weight $\leq 2500$ grams. The sessions took place at three, six, nine and twelve months. Results: Was found that only children in the MP group initiated triadic interactions at nine months $(M=0,16$; $D P=0,40)$. The ability involving person at three months was positively associated with weight at birth $(0,812 ; p<0,05)$, the apgar $5(0,861 ; p<0,05)$. Conclusion: Risk and socioeconomic factors influence the social communicative development of preterms.
\end{abstract}

${ }^{1}$ Esta pesquisa obteve o apoio, por meio da concessão de bolsa de estudos, da Coordenação de Aperfeiçoamento de Pessoal de Nível Superior (Capes). Artigo derivado da dissertação de mestrado de Ivete Furtado Ribeiro Caldas, sob supervisão de Marilice Fernandes Garotti e co-orientação de Aurimery Gomes Chermont, defendida no Programa de Pós-Graduação em Teoria e Pesquisa do Comportamento da Universidade Federal do Pará, em 2014.

${ }_{2}^{2}$ As autoras agradecem às alunas de Iniciação Científica Ana Paula Soares, Lorraine Calandrini e Teresa Rocha, que colaboraram para a coleta de dados desta pesquisa.

${ }^{3}$ Endereço para correspondência: Ivete Furtado Ribeiro Caldas, Universidade Federal do Pará, Avenida Pedro Álvares Cabral, 1879, Residencial Lourdes Caldas, bloco B, ap. 202, Marambaia - Belém - PA - Brasil. CEP: 66113-190. E-mail: ivbeiro@yahoo.com.br. 
Keywords: development; mother-infant interaction; preterm; risk factor, communicative.

FACTORES DE RIESGO Y DESARROLLO SOCIOCOMUNICATIVO EN PREMATUROS

\begin{abstract}
Resumen: Introducción: Los recién nacidos prematuros con bajo peso al nacer pueden tener déficits neurológicos y cambios en el desarrollo de la atención. Interacciones tempranas madre-hijo son importantes para evaluar el proceso de riesgo, comprobado por los cambios en la comunicación no verbal. Meta: Compruebe la relación entre los factores de riesgo, socioeconómico y atención el desarrollo de cada grupo en cuatro etapas de desarrollo. Métodos: Incluyendo I 8 díadas con edad gestacional $<36$ semanas $06 / 07$ y el peso al nacer $\leq 2.500$ gramos. Las sesiones tuvieron lugar en tres, seis, nueve y doce meses. Resultados: Se encontró que sólo los niños del grupo MP empezaron interacciones triádicas a los nueve meses $(M=0,16, S D=0,40)$. Habilidad implica persona a los tres meses se asoció positivamente con el peso al nacer $(0,812 ; p<$ $0,05)$ y apgar5 $(0,86 \mathrm{I} ; \mathrm{p}<0,05)$. Conclusión: Factores de riesgo y socioeconómicos interfieren en el desarrollo atención de los bebés prematuros
\end{abstract}

Palabras clave: desarrollo; interacción madre-hijo; prematuridad; factor de riesgo; comunicativo.

O desenvolvimento sociocomunicativo da criança no primeiro ano de vida envolve várias etapas. Geralmente, os primeiros sinais comunicativos incluem o olhar, o chorar, o vocalizar e o sorrir, bem como movimentos da face, dos lábios e do corpo em resposta à estimulação tátil, vocal ou verbal do cuidador (Seild-de-Moura \& Ribas, 2004). Essas interações diádicas possibilitam trocas denominadas face a face, caracterizadas especialmente pelo contato visual mútuo e pela ocorrência de turnos com sequências comportamentais nas quais o cuidador espera a reação da criança para então responder (Keller, 2007).

A partir dos seis meses, com o aprimoramento das capacidades sensoriais, perceptivas, motoras e sociais, a criança passa a interagir com objetos, agarrando e manipulando-os, e com pessoas, configurando interações denominadas triádicas (criança-objeto-adulto) que se alternam com as diádicas (criança-adulto ou criança-objeto) (Adamson \& Bakeman, 2006). Nesse contexto triádico podem ser observados comportamentos que respondem às tentativas do adulto para iniciar atividades (seguir o apontar e/ou olhar do parceiro social), e, posteriormente, comportamentos que iniciam tais atividades porque direcionam a atenção do adulto para si ou para eventos externos (apontar, mostrar, alternar o olhar entre objeto e adulto) (Oliveira, Zaparolli, Assis, \& Pinheiro, 2013).

O desenvolvimento neuropsicobiológico é influenciado pelo quanto os pais estão dispostos a investir na criança (Keller, 2007), a qualidade da prestação de cuidados precoce parece desempenhar um papel importante na trajetória do desenvolvimento das habilidades sociocomunicativas de neonatos, incluindo os prematuros (Seild-de Moura et al., 2004; Seidl-de-Moura, Bandeira, Campos, da Cruz, Amaral, \& de Marca, 2009). Por sua vez, esse investimento parental está condicionado a fontes ecológicas e sociais disponíveis para os pais (nível socioeconômico, instrução formal, idade dos pais 
ou cuidadores, presença de irmãos e suporte social) e a fatores ligados à criança (saúde, idade, sexo) (Seidl-de-Moura et al., 2009). O desenvolvimento dos neonatos prematuros depende da complexa interação entre esses fatores biológicos e ambientais atuantes no cérebro fisiologicamente imaturo, e diferentes fatores de risco e sociodemográficos podem resultar em alterações sociocomunicativas significativas, tais como menor frequência de contato olho a olho e sorriso social durante as interações com suas mães nos primeiros três meses de vida (Johnson, Hollis, Kochhar, Hennessy, Wolke, \& Marlow, 2010; Moore et al., 1995).

Prematuridade ( $<35$ semanas), baixo peso ao nascer $(<2,5 \mathrm{Kg}$ ) (Johnson et al., 2010), baixo escore no Apgar ( $<7)$ aos 5 minutos (Dodds, Fell, Shea, Armson, Allen, \& Bryson, 2011), icterícia neonatal (Guinchat, Thorsen, Laurent, Cans, Bodeau, \& Cohen, 2012; Johnson et al., 2010) uso de esteroides pré-natais, infecções respiratórias (Guinchat et al., 2012), necessidade prolongada de oxigênio e tempo de internação (Larsson, Eaton, Madsen, Vestergaard, Olesen, Agerbo, Schendel, Thorsen, \& Bo Mortensen, 2005) são considerados fatores de risco associados aos distúrbios sociocomunicativos. Prematuros quando comparados a crianças nascidas a termo apresentam dificuldades em manter a atenção visual antes dos três meses, requerem mais tempo para processar informações sobre os estímulos e, ao final do primeiro ano, mostram menor exploração/manipulação ativa de objetos e decréscimo em atividades conjuntas com a mãe (Moore et al., 1995).

Com relação às características sociodemográficas da família, como renda, escolaridade e idade materna (Bandeira \& Seidl-de-Moura, 2012; Ribas, Seidl-de-Moura, \& Bornstein, 2003), essas são consideradas como riscos para o desenvolvimento sociocomunicativo. Quando comparadas às mães de baixa renda e pouca escolaridade, mães de classe média e com maior nível educacional detém mais informações sobre o desenvolvimento infantil (Seidl-de-Moura et al., 2009) respondem mais rapidamente às vocalizações da criança (Herman, 2002) e apresentam maior frequência de contato face a face e estimulação por objeto (Keller, 2007). Além disso, mães urbanas pertencentes à classe média são caracterizadas tanto por terem menos filhos e mais idade à época do primeiro filho; fatores que propiciam maior investimento no desenvolvimento neuropsicobiológico da prole. Esse investimento parental é refletido na disponibilidade de tempo e atenção exclusiva dedicada à criança (Keller, 2007). A atenção disponibilizada para um único filho apresenta impacto positivo no desenvolvimento, pois o foco não é dividido com os demais filhos (Bandeira et al., 2012).

Assim, prematuros nascidos com peso $\leq 2.500$ gramas podem apresentar déficits decorrentes de comprometimentos neurológicos que podem alterar o desenvolvimento sociocomunicativo (Dodds et al., 2011; Guinchat et al., 2012; Larsson et al., 2005), verificado a partir de padrões de comunicação não verbal alterados, observados nas interações mãe-bebê prematuro desde os primeiros meses de vida (Shah, Robbins, Coelho, \& Poehlmann, 2013). A ordem de nascimento e o número de filhos também podem interferir nesse desenvolvimento (Keller, 2007; Seidl-de-Moura et al., 2009). 
Desta forma, o estudo verificou a relação entre fatores de risco (pré, peri e neonatais), sociodemográficos e desenvolvimento sociocomunicativo de prematuros para cada grupo de díades em quatro momentos do desenvolvimento.

\section{Método}

\section{Participantes}

Trata-se de um estudo prospectivo e longitudinal. Os neonatos nasceram e foram internados na Unidade de Terapia Intensiva Neonatal (Utin) de um hospital privado, localizado em Belém (PA). De 17 mães com neonatos prematuros nascidos entre agosto e novembro de 2012, foi excluída uma, por não comparecer a mais de uma sessão, e outra, por óbito neonatal. Assim, participaram do estudo 15 mães, sendo três mães de gêmeos, e 18 neonatos prematuros, com idade gestacional (IG) < 36 6/7 semanas e peso ao nascer $(\mathrm{PN}) \leq 2.500$ gramas. Foram excluídas mães com diagnóstico de doenças psiquiátricas, residentes fora do no município de Belém e que não aceitaram assinar o Termo de Consentimento Livre e Esclarecido (TCLE), e foram excluídos neonatos com malformações congênitas, síndromes genéticas detectadas no período neonatal, bem como os nascidos ou transferidos de outras instituições. O estudo foi submetido à Plataforma Brasil e aprovado pelo Comitê de Ética em Pesquisa da Fundação Pública Estadual Hospital de Clínicas Gaspar Vianna (Parecer n. 176.898).

As 18 díades formadas foram distribuídas em três grupos com seis díades cada, delimitados de acordo com as características gestacionais e ordem de nascimento do neonato na família. O primeiro grupo (três mães e seis neonatos) era composto por mães primíparas e prematuros gemelares (MPG); o segundo, por mães primíparas (MP) e o terceiro, por mães multíparas (MM).

\section{Instrumentos}

Para a coleta dos dados foram utilizados: 1. Ficha Clínica para a obtenção de informações gestacionais da mãe (doenças obstétricas, complicações no parto, número de gestações, tipo de parto e pré-natal), neonatais (peso ao nascer, idade gestacional, Apgar e sexo) e pós-natais (necessidade de suporte ventilatório, fototerapia e presença de complicações neonatais); 2 . Entrevista Sociodemográfica para informações sobre características individuais da mãe e do cônjuge (idade e estado civil), composição familiar (tipo de família, número de filhos, ordem de nascimento do prematuro e densidade demográfica), escolaridade, renda e ocupação remunerada dos pais; 3 . Protocolo de Observação Mãe-criança para codificar a atenção das crianças durante as interações com suas mães. O protocolo foi baseado nos estados de engajamento proposto por Bakeman \& Adamson (1984), tais como habilidades da criança envolvendo pessoa (HP), envolvendo objeto (HO) ou envolvendo objeto e pessoa simultaneamente (HOP) (ver Quadro 1 para definições). 


\section{Quadro I. Habilidades Sociocomunicativas categorizadas no estudo}

\begin{tabular}{|c|c|}
\hline Habilidades & Definição \\
\hline \multicolumn{2}{|c|}{ Habilidades envolvendo pessoa (HP) } \\
\hline Busca contato físico (Cbc) & $\begin{array}{l}\text { Deslocamento em direção ao cuidador que resulta em } \\
\text { brincadeira, afetividade e/ou proteção por parte do } \\
\text { cuidador. }\end{array}$ \\
\hline Movimentar corpo (Cmovc) & $\begin{array}{l}\text { Movimenta o corpo ou partes dele em resposta ao } \\
\text { comportamento do cuidador. }\end{array}$ \\
\hline Olhar "para” cuidador (Coc) & Olha em direção à face do cuidador. \\
\hline Sorrir "para” cuidador (Cs) & Olha em direção à face do cuidador e sorri. \\
\hline Tocar cuidador (Ct) & $\begin{array}{l}\text { Toca ou encosta no cuidador demonstrando afeto ou } \\
\text { carinho. }\end{array}$ \\
\hline Chorar (Cch) & Contrai a face e emite som de choro. \\
\hline Vocalizar (Cv) & $\begin{array}{l}\text { Emissões vocais da criança, incluindo balbúcio, em } \\
\text { direção ao cuidador. Não eram registrados sons } \\
\text { corporais (tosse, arroto etc.). }\end{array}$ \\
\hline Imitar (Cim) & $\begin{array}{l}\text { Reproduz imediatamente uma ação realizada pelo } \\
\text { cuidador. }\end{array}$ \\
\hline \multicolumn{2}{|c|}{ Habilidades envolvendo objeto $(\mathrm{HO})$} \\
\hline Pegar objetos (Cpo) & $\begin{array}{l}\text { Olha para objeto, pega e o segura, sem envolver } \\
\text { exploração sensorial. }\end{array}$ \\
\hline Movimentar corpo "para” objetos (Cmovo) & $\begin{array}{l}\text { Movimenta o corpo em reposta a um estímulo auditivo } \\
\text { e/ou visual proveniente do objeto. }\end{array}$ \\
\hline Olhar para objeto (Coo) & $\begin{array}{l}\text { Fixa e/ou acompanha o movimento do objeto } \\
\text { movimento com o olhar. }\end{array}$ \\
\hline Manipular objeto (Cmo) & $\begin{array}{l}\text { Olha, pega e manipula o objeto explorando-o } \\
\text { sensorialmente (ex.: exploração oral, bater, girar). }\end{array}$ \\
\hline Sorrir “para” objeto (Cso) & $\begin{array}{l}\text { Sorri em direção ao objeto para o qual está olhando e/ } \\
\text { ou manipulando. }\end{array}$ \\
\hline \multicolumn{2}{|c|}{ Habilidades envolvendo objeto e pessoa (HOP) } \\
\hline Apontar (Capo) & $\begin{array}{l}\text { Estende o braço e o dedo indicador apontando para um } \\
\text { objeto, pessoa ou evento tendo como resultado o início } \\
\text { de uma atividade conjunta com o cuidador. }\end{array}$ \\
\hline Iniciar interação triádica (Ciit) & $\begin{array}{l}\text { Comportamentos emitidos em direção ao cuidador } \\
\text { (p. e., dar/mostrar objetos, alternar olhar entre objeto } \\
\text { e cuidador) que resultam em atividades conjuntas sobre } \\
\text { um mesmo objeto ou evento. }\end{array}$ \\
\hline $\begin{array}{l}\text { Comportamento de solicitação } \\
\text { (CS) }\end{array}$ & $\begin{array}{l}\text { Utiliza os mesmos comportamentos que iniciam } \\
\text { atividades conjuntas (apontar, alternar olhar entre } \\
\text { objeto e cuidador) apenas para requerer algo. Nenhuma } \\
\text { interação é estabelecida depois da obtenção do objeto. }\end{array}$ \\
\hline
\end{tabular}

Fonte: Elaborado pelas autoras. 


\section{Procedimento}

Depois dos esclarecimentos sobre o objetivo da pesquisa e assinatura do Termo de Consentimento Livre e Esclarecido (TCLE), estabelecia-se um cronograma de sessões para os 12 meses subsequentes à alta hospitalar. Nesse momento, também, as mães respondiam às perguntas do experimentador referente à Ficha Clínica e a Entrevista Sociodemográfica. Trimestralmente, as sessões ocorreram ao longo do primeiro ano de vida, equivalendo aos três, seis, nove e doze meses de idade (Idade Cronológica). Porém, em virtude de a amostra ser composta por prematuros, fez-se necessário o cálculo da Idade Corrigida (ICg), que visou adequar o desenvolvimento das crianças para cada idade registrada (BRASIL, 2012). Assim, as ICg corresponderam em média, respectivamente, a um, quatro, sete e dez meses.

As sessões foram desenvolvidas de forma semiestruturada, no horário diurno, e registradas em vídeo por uma aluna de iniciação científica treinada. As sessões foram realizadas em um consultório no mesmo hospital em que os prematuros nasceram, durante a consulta de follow up do prematuro, e dependendo da idade da criança, diferentes brinquedos foram selecionados. As observações mãe-criança foram integralmente registradas em vídeo para posterior categorização das habilidades sociocomunicativas.

Cada sessão durava trinta minutos, divididos em três etapas de 10 minutos cada. Na primeira, eram realizados o acolhimento da díade e o registro de informações e intercorrências clínicas ocorridas no trimestre anterior; na segunda, solicitava-se às mães que interagissem livremente com seus filhos; e na última, era o experimentador quem interagia. Entretanto, para a análise dos dados foi utilizado apenas o período de interação livre mãe-criança.

\section{Análise dos dados}

Em cada sessão de interação livre mãe-criança, os seis minutos finais dos vídeos foram transcritos e analisados quantitativamente pelo aplicativo Transana 2.53 (www. transana.org) para registro da duração e frequência com que as díades, para cada idade, iniciavam e/ou respondiam às interações. As diferenças entre os grupos foram verificadas pelo teste de Kruskal-Wallis (X2) e as intragrupos pelo teste de Fridman. As correlações entre as variáveis sociodemográficas (renda familiar, escolaridade e idade materna), fatores de risco e habilidades sociocomunicativas foram verificadas pelo teste de Spearman. As análises estatísticas foram realizadas pelo Statistical Package for Social Science (SPSS) versão 18.0. O índice de concordância entre dois observadores independentes, previamente treinados, foi calculado com base em $35 \%$ do total das sessões. O índice de concordância foi de $83 \%$ nas habilidades envolvendo pessoa (HP), $87 \%$ nas envolvendo objeto (HO) e $100 \%$ nas envolvendo objeto e pessoa (HOP).

\section{Resultados}

A Tabela 2 detalha as características sociodemográficas das famílias e os aspectos clínicos das díades para cada grupo. Em relação à escolaridade materna, 83,3\% das 
mães do grupo MP tinham ensino superior completo e 66,7\% das mães do grupo MPG tinham renda familiar entre $\mathrm{R} \$ 2.500,00$ e $\mathrm{R} \$ 4.999,00$. Quanto à Idade Gestacional, todas as mães do grupo MPG tiveram seus filhos entre $31-33$ semanas e $100 \%$ das crianças dos grupos MPG e MP nasceram com peso entre 1.000 e 1.499 gramas. Além disso, 83,3\% dos neonatos dos grupos MPG e MP fizeram uso de oxigenoterapia entre 3 a 10 dias e todos os neonatos do grupo MM apresentaram infecção neonatal durante a internação.

Tabela 2. Características sociodemográficas das famílias e aspectos clínicos das díades para cada grupo

\begin{tabular}{|c|c|c|c|}
\hline & MPG $(\mathrm{N}=6)^{*}$ & $M P(N=6)$ & $M M(N=6)$ \\
\hline Variáveis & $\%$ & $\%$ & $\%$ \\
\hline \multicolumn{4}{|l|}{ Escolaridade materna } \\
\hline Superior completo & 66,7 & 83,3 & 33,3 \\
\hline Médio completo & 33,3 & 16,7 & 50 \\
\hline Médio incompleto & 0 & 0 & 16,7 \\
\hline \multicolumn{4}{|l|}{ Idade materna (anos) } \\
\hline $22-30$ & 33,3 & 50 & 33,3 \\
\hline $31-40$ & 66,7 & 50 & 66,7 \\
\hline \multicolumn{4}{|l|}{ Renda familiar (reais) } \\
\hline $2.500-4.999$ & 66,7 & 33,3 & 50 \\
\hline $5.000-7.499$ & 33,3 & 50 & 50 \\
\hline$>10.000$ & 0 & 16,7 & 0 \\
\hline \multicolumn{4}{|l|}{$\begin{array}{l}\text { Idade gestacional } \\
\text { (semanas) }\end{array}$} \\
\hline $31-33$ & 100 & 83,3 & 66,7 \\
\hline $34-36$ & 0 & 16,7 & 33,3 \\
\hline \multicolumn{4}{|c|}{ Peso ao nascer (gramas) } \\
\hline $1000-1499$ & 0 & 0 & 33,3 \\
\hline $1500-1999$ & 100 & 100 & 66,7 \\
\hline \multicolumn{4}{|l|}{ Sexo } \\
\hline Masculino & 33,3 & 50 & 33,3 \\
\hline Feminino & 66,7 & 50 & 66,7 \\
\hline \multicolumn{4}{|l|}{ Dias de internação } \\
\hline $10-20$ dias & 66,8 & 66,8 & 50 \\
\hline $21-30$ dias & 16,6 & 16,6 & 33,3 \\
\hline $31-42$ dias & 16,6 & 16,6 & 16,7 \\
\hline
\end{tabular}


Tabela 2. Características sociodemográficas das famílias e aspectos clínicos das díades para cada grupo (conclusão)

\begin{tabular}{lccc}
\hline & $M P G(N=6)^{*}$ & $M P(N=6)$ & $M M(N=6)$ \\
\cline { 2 - 4 } Variáveis & $\%$ & $\%$ & $\%$ \\
\hline 5 a 7 & 16,7 & 16,7 & 0 \\
8 a 10 & 83,3 & 83,3 & 100 \\
Dias em oxigenoterapia & & & 66,7 \\
3 a 10 & 83,3 & 83,3 & 33,3 \\
II a 25 & 16,7 & 16,7 & \\
Icterícia & & & 83,3 \\
Sim & 100 & 66,6 & 100 \\
Infecção neonatal & & & \\
Sim & 83,3 & 66,6 & \\
\hline
\end{tabular}

* Esse grupo era formado por três mães de gêmeos

Fonte: Elaborada pelas autoras.

\section{Duração das habilidades sociocomunicativas nos grupos}

Ao analisar o percentual de duração das três habilidades HP, HO e HOP observadas nos grupos MP, MPG e MM ao longo das idades, pode-se verificar que em todos os grupos houve predomínio de HP aos três meses, variando de $90,10 \%$ a $98,68 \%$. A HO apresentou aumento aos seis meses em todos os grupos, variando de $71,22 \%$ a $80,13 \%$. Foi registrada $\mathrm{HOP}(0,13 \%)$ aos seis meses no grupo $\mathrm{MPG}$, diferente dos demais grupos que esteve presente somente a partir dos nove meses. O percentual de duração de HOP variou de $1,63 \%$ a $3,88 \%$ aos nove meses e de $3,23 \%$ a 3,53\% aos doze meses. Os resultados indicaram que não houve diferença significativa entre os grupos na duração das habilidades nas diferentes idades.

Para análise intragrupo, foi constatado que o grupo MP não apresentou diferença significativa na duração das habilidades nas diferentes idades. Já no grupo MPG foram observadas diferenças significativas nas HP $(x 2=11,00 ; p=0,012)$, com maior duração aos três meses $(M=90,70 ; D P=16,10)$. Nas HO, também foram observadas diferenças significativas $(x 2=11,00 ; p=0,012)$, com aumento na duração aos seis meses $(M=74,38 ; p=24,66)$. Nas HOP, também foram observadas diferenças significativas $(x 2=10,35 ; p=0,01)$, verificando episódios triádicos aos seis meses $(M=0,13$; $D P=0,32)$. No grupo $M M$, também foram observadas diferenças significativas nos estados de HP $(x 2=13,40 ; p=0,04)$, com maior duração aos três meses $(M=98,68 ; p=2,48)$. Nas HO, foram observadas diferenças significativas ( $x 2=15,00 ; p=0,002)$, com aumento na duração aos seis meses ( $M=71,21$; $\mathrm{DP}=29,5)$. Em relação às HOP, não houve diferença significativa nas diferentes idades.

\section{Trajetória de desenvolvimento das habilidades sociocomunicativas}

As crianças de todos os grupos apresentaram padrões similares de desenvolvimento das habilidades sociocomunicativas (Quadro 1). Entre os três e seis meses, a frequên- 
cia de HP diminuiu, enquanto HO aumentou. Apenas a partir dos seis meses é que as interações triádicas (criança-objeto-mãe) foram observadas. Os grupos diferiam com relação à frequência e ao tipo de comportamento sociocomunicativo observado. Para os grupos MPG e MM, todas as habilidades comunicativas envolvendo pessoa diminuíram e permaneceram próximas de zero a partir do sexto mês. Por outro lado, as crianças do grupo MP mantiveram a vocalização $(M=5,5 ; D P=1,87)$ e o olhar para a mãe $(M=5,83 ; D P=2,33)$ como principais formas comunicativas aos 9 e 12 meses.

Nas HO, as crianças de todos os grupos apresentaram maior frequência em olhar para o objeto a partir dos seis meses, destacando as crianças do grupo MP quando comparadas com as demais ( $M=10,83 ; 7,33$ e 8,5 aos 6,9 e 12 meses, respectivamente), que também apresentavam maior frequência em manipulação de objetos em todas as idades. Para o grupo MM, a manipulação aumentou enquanto o pegar diminuiu aos 12 meses. As crianças de MPG pegavam objetos, mas só os manipulavam aos 12 meses $(M=0,5 ; D P=0,83)$. Quanto às HOP, somente as crianças do grupo MP iniciaram interações triádicas (Ciit) aos $9(M=0,16$; $D P=0,40)$ e aos 12 meses $(M=1,66$; DP $=0,51$ ). O apontar para compartilhar foi observado nas crianças do grupo MPG aos 9 meses $(M=0,16$; $D P=0,40)$, nas do $M P$, aos $12(M=0,33$; $D P=0,51)$ e não foi registrado para nenhuma criança do MM. O comportamento de solicitação (CS) foi observado no grupo MP desde os 6 meses $(M=0,16$; $D P=0,40)$ no $M M$, apenas aos $12(M=0,5$; $0,83)$ e em MPG a partir dos $9(M=0,33 ; D P=0,51)$ e foi a única habilidade observada para esse grupo aos 12 meses.

\section{Relações entre características sociodemográficas, fatores de risco e habilidades sociocomunicativas}

Quanto às características sociodemográficas, apenas a idade materna estava associada às habilidades sociocomunicativas. Aos 9 meses a idade materna estava associada negativamente à $\mathrm{HP}(-0,506 ; p<0,01)$ e positivamente à $\mathrm{HO}(0,504 ; p<0,05)$. Esses resultados sugerem que mães mais jovens tendem a estimular mais habilidades envolvendo pessoas, enquanto aquelas com mais idade priorizam o envolvimento com objeto.

$\mathrm{Na}$ análise entre habilidades sociocomunicativas e fatores de risco (IG, PN, dias de internação, Apgar5, dias em oxigenoterapia, presença de icterícia neonatal, de infecção neonatal e uso de esteroide antenatal), houve associação negativa entre infecção neonatal e $\operatorname{HOP}(-0,542 ; p<0,05)$ aos 6 meses de idade. Nenhuma outra associação foi encontrada. Entretanto, ao analisar separadamente os grupos, foram observadas relações significativas entre habilidades sociocomunicativas e fatores de risco somente no grupo MP (Tabela 3). Nesse grupo, a HP aos 3 meses estava positivamente associada ao peso da criança ao nascer $(0,812 ; p<0,05)$, ao apgar $5(0,861 ; p<0,05)$ e ao uso de esteroide antenatal $(0,878 ; p<0,05)$. Associações negativas foram observadas aos 12 meses entre HP e infecção neonatal $(-0,840 ; p<0,05)$, e entre HO e o Apgar 5 $(-0,926 ; p<0,01)$. 


\section{Discussão}

Os resultados mostram que não houve diferença significativa entre os grupos na duração das habilidades, talvez por ser tratar de uma amostra homogênea e com características similares. Entretanto, diferenças na duração das habilidades sociocomunicativas foram verificadas ao longo das idades, conforme descrito por Bakeman et al. (1984) que descreve o modelo hierárquico de desenvolvimento da atenção da criança, evoluindo de diádico a triádico. Além do mais, a literatura descreve apenas observações em situação natural (casa) (Keller, 2007), não encontrado observações realizadas em ambiente semiestruturado (consultório), portanto, um dos diferenciais desse estudo.

Comparando crianças nascidas a termo e prematuros que foram submetidos a fatores de risco pré, peri e neonatais, estudos mostram que os prematuros representam uma população vulnerável a alterações sociocomunicativas, pois apresentam risco aumentado para alterações neurocomportamentais, incluindo alterações cognitivas, dificuldade de aprendizagem e maiores problemas comportamentais. O "pobre" processamento cognitivo pode resultar em dificuldades sociais e de atenção. Déficits cognitivos e comportamentais ocorrem em 25 a $50 \%$ dos neonatos nascidos prematuros (Shah et al., 2013).

Em relação às HP, é importante ressaltar o seu predomínio em todos os grupos aos 3 meses. Nesse período é que as primeiras interações são estabelecidas entre a mãe e o bebê, evidenciadas pela presença do olhar, do vocalizar e do movimentar em direção à mãe. Essas habilidades da criança são dirigidas à mãe que os recebe e, simultaneamente, dirige ao bebê com sons e gestos. Contudo, o grupo MM destacou-se na sua duração de HP em relação aos demais grupos. Uma hipótese pode ser baseada no estudo de Bandeira et al. (2012) que afirmam que quanto menor o filho, mais as mães valorizam o investimento em cuidados, como banho, troca de fraldas, amamentação, estimulando maior envolvimento da criança com pessoa.

Aos 6 meses HP foram reduzidas, e as interações mostraram-se mais complexas. Nessa idade, uma maior variedade de HO estava presente, corroborando Adamson et al. (2006) e Mundy e Newell (2007). Para Tomasello, Carpenter, Call, Behne e Moll (2005), essas habilidades são importantes para o desenvolvimento da atenção coordenada que vai surgir alguns meses mais tarde. O grupo MPG merece destaque pela presença de episódios envolvendo não só objeto, mas também pessoa, aos 6 meses. Hipotetiza-se que, como as mães desse grupo tinham apenas um filho, o incentivo e a atenção disponibilizada eram maiores, podendo justificar a presença dessas interações triádicas mais precoces, quando comparado aos demais grupos.

Dos 9 e 12 meses, todos os grupos apresentaram HOP, porém, com durações muito curtas comparando com crianças com desenvolvimento típico. Além do mais, aos 9 meses, as crianças do grupo MP apresentaram menor duração HOP do que as crianças dos demais grupos. Nossa hipótese é que nesse grupo, como os filhos eram gêmeos, a mãe precisava dividir a atenção com duas crianças da mesma idade e que apresentavam praticamente as mesmas necessidades. Isso reforça a ideia de que a mãe funciona 
como um sistema de suporte social para o desenvolvimento da criança (Aquino \& Salomão, 2011), pois em muitos episódios ela interrompia a interação com um filho para atender às necessidades do outro, principalmente nos casos de choro e fome.

Em relação à frequência das HP, observaram-se aos 3 meses predomínio do olhar em direção ao cuidador (Coc); movimento quando olhava para o cuidador (Cmovc) e vocalização (Cv) tanto nas crianças do grupo MP quanto nas do MGP. Seidl-de-Moura et al. (2004) também mostraram a predominância do vocalizar e do olhar para a mãe. Adicionalmente, o predomínio do chorar (Cch) no grupo MM deve ser considerado. Supõe-se que, como as mães dos grupos MP e MPG eram primíparas, elas disponibilizavam a atenção mais imediatamente às necessidades dos seus filhos do que as MM que precisavam dividir atenção com outros filhos de outras idades. Quanto ao vocalizar (Cv), tanto o grupo MPG quanto o MM apresentaram declínio na frequência ao longo das idades, apenas no MP apresentou aumento a partir dos 9 meses. Essa frequência reduzida do vocalizar aos 12 meses também foi descrita por Garner, Landry e Richardson (1991) que compararam o vocalizar de neonatos a termo $(M=23,67$; $D P=20,80)$ e prematuros submetidos a fatores de risco $(M=21,66 . D P=13,50)$.

Já nas HO foram observados o predomínio do agarrar objeto (Cao); o olhar para o objeto (Coo) e a tentativa de pegá-lo (Cpo) em todos os grupos, a partir dos 6 meses. Corroborando Aquino et al. (2011) que encontraram aos 6 meses maior frequência do levantar ou dar os braços para o adulto, inclinar o corpo em direção a objetos, olhar fixamente para objetos e estender os braços em sua direção. Todavia, as crianças do grupo MP destacaram-se por apresentar maior frequência de Coo e Cmo dos 6 aos 12 meses, reforçando a ideia de maior disponibilidade das suas mães em estimulá-los por serem filhos únicos.

Considerando as HOP dos 9 aos 12 meses, as mais frequentes foram iniciar comportamento de solicitação (ICS), iniciar interação triádica (Ciit) e apontar (Capo). Ao final do primeiro ano de vida, estudos apontam que neonatos a termo e com desenvolvimento típico já começam a exibir o mostrar e o apontar (Adamson et al., 2006; Aquino et al., 2011). Entretanto, por se tratar de crianças prematuras e que foram expostas a fatores de riscos, houve baixa frequência de habilidades triádicas, principalmente a partir dos 9 meses. Ao comparar os grupos, as crianças de MP apresentaram maior frequência de episódios triádicos, sugerindo que a menor exposição desse grupo aos fatores de risco (maior média de peso ao nascer, 50\% das mães realizaram esteroides antenatais e menor presença de infecção neonatal) possa ter contribuído para esses resultados.

Dentre as variáveis sociodemográficas analisadas, a idade materna apresentou relação significativa. A influência da idade materna tem relação direta na intensidade e no tipo da estimulação dos seus filhos. As mães mais velhas apresentam maior investimento na prole que as mais nova porque apresentam menos chances de produzir novos descendentes, além do que as mães mais velhas utilizam mais os objetos para intermediar as interações do que as mais novas, que priorizam o face a face (Keller, 2007). 
Portanto, os achados evidenciam que tanto os fatores de risco quanto os sociodemográficos influenciam o desenvolvimento das habilidades sociocomunicativas das crianças nascidas prematuras, contribuindo para os estudos de interações mãe-bebê. Entretanto, não houve a participação de neonatos a termo (Grupo Controle), sendo considerada uma limitação do estudo. Futuras pesquisas merecem novas análises com maiores amostras, contextos culturais e níveis socioeconômicos diferentes, com o intuito de identificar outras diferenças nas interações. Chama-se atenção também para os profissionais envolvidos no cuidado desses prematuros para estimularem o seu desenvolvimento sociocomunicativo, respeitando sempre suas limitações comportamentais.

\section{Referências}

Adamson, L. B., \& Bakeman, R. (2006). Developmental of displaced speech in early-mother-child conversations. Child Development, 77(1), 186-200.

Aquino, F. S. B., \& Salomão, N. M. R. (2011). Habilidades sociocomunicativas de bebês no primeiro ano de vida: um estudo longitudinal. Paidéia, 21(50), 335-344.

Bandeira, T. T. A., \& Seild de Moura, M. L. (2012). Crenças de pais e mães sobre investimento parental. Paidéia, 22(53), 355-363.

Bakeman, R., \& Adamson, L. B. (1984). Coordinating attention to people and objects in mother-infant and peer-infant interation. Child Development, 55(4), 1278-1289.

Brasil (2012). Manual AIDPI neonatal (3a ed). Brasília: Ministério da Saúde. (Série Normas e Manuais Técnicos).

Dodds. L., Fell, D. B., Shea, S., Armson, B. A., Allen, A. C., \& Bryson, S. (2011). The role of prenatal, obstetric and neonatal factors in the development of autism. Journal of Autism and Developmental Disorders, 41(7), 891-902.

Garner, P. W., Landry, S. H., \& Richardson, M. A. (1991). The development of joint attention skills in very-low-birth-weight infants across the first 2 years. Infant behavior and development, 14(4), 489-495.

Guinchat. V., Thorsen. P., Laurent. C., Cans, C., Bodeau, N., \& Cohen, D. (2012). Pre, peri- and neonatal risk factors for autism. Acta Obstetriciaet et Gynecologica Scandinavica, 91(3), 287-300.

Herman, R. (2002). Characteristic developmental patterns of language and communication in hearing and deaf babies 0-2 years. Department of Language and Communication Science, 16(4), 56-63.

Johnson, S., Hollis, C., Kochhar, P., Hennessy, E., Wolke, D., \& Marlow, N. (2010). Autism spectrum disorders in extremely preterm children. The Journal of Pediatrics, 156(4), 525-531. 
Keller, H. (2007). Cultures of infancy. Mahwah: Lawrence Erlbaum.

Larsson, H. J., Eaton, W. W., Madsen, K. M., Vestergaard, M., Olesen, A. V., Agerbo, E., Schendel, D., Thorsen, P., \& Bo Mortensen, P. (2005). Risk factors for autism: perinatal factors, parental psychiatric history, and socioeconomic status. Am J Epidemio, 161(10), 916-925.

Moore, C., \& Dunham, P. J. (1995). Joint Attention. Its origins and role in development. In Landry, S. H. (Ed.). The development of joint attention in premature low birth weight infants: Effects of early medical complications and maternal attention-directing behaviors (pp. 223-249). Hillsdale: Lawrence Erlbaum Associates.

Mundy, P., \& Newell, L. (2007). Attention, joint attention and social cognition. Association for Psychological Science, 16(5), 269-274.

Oliveira, A. I. A., Zaparolli, D. A., Assis, G. J. A., \& Pinheiro, M. A. (2013). Desenvolvimento humano: contribuições para a aprendizagem e a inclusão social. In M. F. Garotti, A. C. Santos, I. F. Ribeiro, A. I. A. Oliveira, \& S. S. C. Silva (1a ed.). Alterando rotas alteradas? Detecção precoce de riscos e prevenção em transtornos do espectro do autismo (pp.51-66). Belém: EDUEPA.

Ribas Jr., R. C., Seidl-de-Moura, M. L., \& Bornstein, M. H. (2003). Socioeconomic status in brazilian psychological research: socioeconomic status and parenting knowledge. Estud Psicol (Natal), 8(3), 385-392.

Seidl-de-Moura, M. L. (2004). O bebê do século XXI e a psicologia em desenvolvimento. In Seidl de Moura, M. L., \& Ribas, A. F. P. (Eds.). Evidências sobre características de bebês recém-nascidos: um convite para reflexões teóricas (pp. 21-59). São Paulo: Casa do Psicólogo.

Seidl-de-Moura, M. L., Bandeira, T. T. A., Campos, K. N., da Cruz, E. M., Amaral, G.S., \& de Marca, R. G. C. (2009). Parenting cultural models of a group of mothers from Rio de Janeiro. The Spanish Journal of Psychology, 12(2), 506-517.

Shah, P. E., Robbins, N., Coelho, R. B., \& Poehlmann, J. (2013). The paradox of prematurity: the behavioral vulnerability of late preterm infants and the cognitive susceptibility of very preterm infants at 36 months post-term. Infant Behavior \& Development, 36(1), 50-62.

Tomasello, M., Carpenter, M., Call, J., Behne, T., \& Moll, H. (2005). Understanding and sharing intentions: The origins of cultural cognition. Behavioral and Brain Sciences, 28(5), 675-691.

Submissão: 2.2.2015

Aceitação: 15.3.2016 